\title{
Rauchen während der Schwangerschaft - Risiko für intrauterine Wachstumsrestriktion und bleibende Kleinwüchsigkeit
}

\section{Smoking during Pregnancy - Risk for Intrauterine Growth Retardation and Persisting Microsomia}

\author{
Autoren \\ S. Koch ${ }^{1}$, C. Vilser ${ }^{2}$, W. Groß ${ }^{3}$, E. Schleußner ${ }^{4}$ \\ Institute \\ ${ }^{1}$ Klinik für Frauenheilkunde und Geburtshilfe, Thüringen-Kliniken „Georgius-Agricola“ Saalfeld-Rudolstadt \\ ${ }^{2}$ Klinik für Kinder- und Jugendmedizin, Universitätsklinikum Jena, Friedrich-Schiller-Universität Jena \\ ${ }^{3}$ Klinik für Frauenheilkunde und Geburtshilfe, SRH Klinikum Gera \\ ${ }^{4}$ Abteilung Geburtshilfe, Klinik für Frauenheilkunde und Geburtshilfe, Universitätsklinikum Jena, \\ Friedrich-Schiller-Universität Jena
}

Schlüsselwörter
Rauchen
Schwangerschaft
small for gestational age
Kleinwuchs
Key words
smoking
pregnancy
small for gestational age
microsomia

eingereicht $\quad 21.12 .2011$ angenommen $\quad 01.03 .2012$ nach Überarbeitung

\section{Bibliografie}

DOI http://dx.doi.org/ 10.1055/s-0032-1308958

Z Geburtsh Neonatol 2012; 216: 77-81

(c) Georg Thieme Verlag KG Stuttgart · New York ISSN 0948-2393

Korrespondenzadresse Prof. Dr. med. Ekkehard Schleußner

Abteilung Geburtshilfe Klinik für Frauenheilkunde und Geburtshilfe

Bachstraße 18 07743 Jena

Tel.: $+49 / 03641 / 933230$ Fax: $+49 / 03641 / 933486$ Ekkehard.Schleussner@med. uni-jena.de

\section{Zusammenfassung}

\section{$\nabla$}

Zielstellung: Rauchen während der Schwangerschaft ist ein wesentlicher Risikofaktor für eine intrauterine Wachstumsrestriktion. Ziel der vorgestellten Thüringer SGA-(small-for-gestational-age)-Studie ist den Einfluss des Nikotinkonsums während der Schwangerschaft auf das Geburtsgewicht und -größe sowie die postnatale Wachstumsdynamik und das Aufholwachstum von SGA-Kindern zu untersuchen.

Methode: Von 1992-2002 wurden in den Perinatalzentren Jena und Gera 2447 lebendgeborene Kinder mit einem Geburtsgewicht (GG) $<10$. Perz. u/o einer Geburtslänge $(G L)<-2,0$ SDS ermittelt. Die Eltern der 383 schwer wachstumsretardierten SGA-Kinder (GG u/o GL <-2,5 SDS) wurden angeschrieben und um Übermittlung der aktuellen Größe und des Gewichts ihres Kindes gebeten, wobei wir 108 Antworten erhielten (Alter 8,0 $\pm 3,4$ Jahre). Ergebnisse: Der Anteil der SGA-Geburten bezogen auf alle Lebendgeburten hat von $1992 \mathrm{mit}$ $14,1 \%$ bis 2002 mit $9,4 \%$ abgenommen. $14 \%$ dieser Kinder kamen als Frühgeburten zur Welt. Der Zigarettenkonsum lag im Mittel bei 2 Zigaretten pro Tag (range 0-40). Im SGA-Kollektiv gaben $17,6 \%$ der Mütter an während der Schwangerschaft geraucht zu haben, bei den schwer wachstumsretardierten Kindern 26,9\%. Es besteht eine negative Korrelation von Zigarettenkonsum und dem Geburtsgewicht $(r=-0,09 ; p<0,01)$ und der Geburtslänge $(r=-0,08 ; \quad p<0,01) .30,6 \%$ der ehemals schwer wachstumsretardierten Kinder zeigten kein Aufholwachstum, wobei SGA-Kinder von Raucherinnen ein doppelt so hohes Risiko für einen Kleinwuchs hatten.

Schlussfolgerung: Der Nikotinkonsum während der Schwangerschaft stellt einen Risikofaktor für das Auftreten einer SGA-Geburt dar und kann zu einer über die Schwangerschaft hinaus reichenden bleibenden Beeinflussung des kindlichen Wachstumsverhaltens mit Ausbleiben des Aufholwachstums führen.

\section{Abstract}

$\nabla$

Background: Smoking during pregnancy is a major risk factor for intrauterine growth retardation. The aim of the Thuringian SGA - (small-forgestational-age) - study was to evaluate the effects of maternal smoking during pregnancy on birth weight and length as well as postnatal growth dynamics and catch-up growth.

Methods: Between 1992 and 2002 in all 2447 liveborn children were assessed with birth weight (GG) $<10^{\text {th }}$ percentile and/or birth length $(\mathrm{GL})<-2.0$ SDS. A questionnaire was sent to 383 parents of severe SGA children (GG and/or $\mathrm{GL}<-2.5$ SDS) to report weight and height of the children actually. 108 reports could analysed (mean age $8.0 \pm 3.4$ years of life).

Results: The number of SGA babies in regard to all liveborn children decreased from $14.1 \%$ to $9.4 \%$ between 1992 and $2002.14 \%$ of SGA babies were born preterm. The mean nicotine abuse was 2 cigarettes per day (range $0-40$ ). $17.6 \%$ of the mothers of SGA babies were smoking, whereas in severe SGA $26.9 \%$ of smokers was recorded. There is a inverse correlation of nicotine abuse with birth weight $(r=-0.09 ; \mathrm{p}<0.01)$ or birth length $(\mathrm{r}=-0.08 ; \mathrm{p}<0.01)$. Catch-up growth did not exist in $30.6 \%$ of the severe growth restricted children. The risk for short stature in later life was doubled in SGA children.

Conclusions: Nicotine abuse during pregnancy is a risk factor for an SGA baby and could have long-lasting effects on growth dynamics during childhood with a lack of catch-up growth. 


\section{Einführung \\ $\nabla$}

Eine intrauterine Wachstumsrestriktion (IUWR) kann heute mithilfe der Möglichkeiten der pränatalen Ultraschalldiagnostik anhand populationsbasierter Perzentilenkurven des fetalen Wachstums mit großer Zuverlässigkeit frühzeitig festgestellt werden [1].

Im Gegensatz zu den diagnostischen Möglichkeiten sind die einer vorgeburtlichen Therapie nach wie vor eingeschränkt, sodass diese Kinder intrauterin ungünstigen Entwicklungsbedingungen ausgesetzt sind, die zu einer pränatalen Fehlprogrammierung fetaler Regulationssysteme führen und auch als SGA (small-for-gestational-age) Kinder zur Welt kommen [2].

Von wesentlicher Bedeutung sind deshalb Risikofaktoren, welche zu einer intrauterinen Wachstumsrestriktion führen und deren Prävention. Rauchen während der Schwangerschaft stellt einen der wichtigsten Risikofaktoren dar, der mit bis zu 40\% der Fälle von Wachstumsrestriktion assoziiert ist [3].

Bislang liegen jedoch keine Untersuchungen vor, die das Wachstumsverhalten nach der Geburt dieser Kinder beschreiben. Ziel unserer Studie ist daher den Einfluss von Rauchen während der Schwangerschaft nicht nur auf das intrauterine Wachstum, sondern auch die postnatale Entwicklung der wachstumsretardiert geborenen Kinder zu untersuchen.

\section{Patienten und Methoden}

$\nabla$

Anhand der perinatologischen Basiserhebungsbögen der Universitätsfrauenklinik Jena und der Frauenklinik Gera wurden alle Lebendgeborenen der Geburtenjahrgänge 1992-2002 mit einer intrauterinen Wachstumsrestriktion ermittelt. Die Kategorisierung erfolgte anhand der tragzeitbezogenen Perzentilenkurven für Geburtsgewicht und Geburtslänge in Mitteleuropa nach Voigt [4]. Eingeschlossen wurden alle lebend geborenen Kinder aus einer Einlingsschwangerschaft mit einem Geburtsgewicht (GG) unterhalb der 10. Perzentile und/oder einer Geburtslänge (GL) kleiner als 2 Standardabweichungen unter der Norm. Ausschlusskriterium war ein mütterlicher Kleinwuchs (Körpergröße $<145 \mathrm{~cm}$ ) sowie eine nicht-kaukasische Ethnizität.

2447 der 23321 durchsuchten Datensätze erfüllten diese Kriterien. Die Eltern von 383 schwer wachstumsretardiert geborenen Kindern mit einem GG und/oder einer GL $\leq-2,5$ SDS (standard deviation score, SDS=(Istgröße - altersgemäße Sollgröße)/altersgemäße Standardabweichung, Referenz nach Lawrence et al. [5]) wurde angeschrieben und um Angabe der aktuellen Größe, des aktuellen Gewichts ihres Kindes sowie ihrer eigenen Körpergrößen gebeten. Letztendlich erhielten wir aktuelle Angaben von 108 Kindern (Alter 8,0 3,4 Jahre). Mithilfe der Größe sowie dem Zeitpunkt der jeweiligen Messung wurden die Kinder den aktuellen Wachstumskurven nach Hesse et al. zugeordnet und die aktuelle Größe in Einheiten der Standardabweichung (SDS) berechnet [6,7].

Die Datenanalyse und statistische Auswertung wurde mit dem Programm SPSS mittels Linearer Korrelationsanalyse, LeveneTest, t-Test n. Student bzw. Welch-Test durchgeführt.

\section{Ergebnisse}

$\nabla$

Von 1992 bis 2002 wurden in der Universitätsfrauenklinik Jena und der Frauenklinik Gera 23321 Kinder geboren, darunter
2447 lebend geborene SGA-Kinder mit Geburtsmaßen kleiner der 10. Perzentile. Deren prozentualer Anteil von 10,5\% entspricht der deutschen Gesamtpopulation [1]. 383 Kinder (1,6\%) waren schwer wachstumsretardiert <2 SDS. Der Anteil der SGAGeburten bezogen auf alle Geburten hat von 1992 mit 14,1\% bis 2002 mit 9,4\% abgenommen ( $\bullet$ Abb. 1). Im SGA-Kollektiv war die Geschlechtsverteilung ausgeglichen (50,1\% Mädchen, 49,9\% Jungen), während in der Gruppe der schwer wachstumsretardierten Kinder 214 Mädchen (55,9\%) gegenüber 169 Jungen $(44,1 \%)$ dominierten. Die Schwangerschaftsdauer betrug in der SGA-Gruppe im Mittel 39,2 $\pm 2,1$ SSW [24-44 SSW] und bei den schwer retardierten Kindern 38,2 $\pm 2,1$ SSW [29-42 SSW]. Der Anteil der Frühgeborenen lag in der SGA-Gruppe bei 14,0\% und bei den schwer wachstumsretardierten SGA-Kindern bei $29,0 \%$. Das Alter der Mütter zur Geburt unterschied sich im gesamten SGA-Kollektiv mit 26,8 $\pm 5,2$ Jahre [14-44 Jahre] nicht von dem der Mütter von schwer retardierten Kindern $(26,9 \pm 5,4$ Jahre [17-44 Jahre]). Auffällig war, dass im Verlauf des Beobachtungszeitraumes das mittlere Alter der Mütter von 25,2 Jahren in 1992 auf 27,3 Jahre in 2002 anstieg ( $\bullet$ Abb. 2). Dieser Trend findet sich auch im maternalen Durchschnittsalter aller Geburten in Thüringen, wobei sich kein wesentlicher Unterschied zum Beobachtungskollektiv darstellte (Daten vom Statistischen Landesamt Thüringen) [8]. Je älter die Mütter waren, desto weiter wich das Geburtsgewicht, nicht jedoch die Geburtslänge von der Norm ab $(r=-0,05, p<0,05)$.

Während der Schwangerschaft rauchten 17,6\% der Mütter aus der SGA-Gruppe und 26,9\% der Mütter mit schwer retardierten Kindern. Es zeigten sich signifikant niedrigere somatische Geburtsparameter bei den Neugeborenen rauchender Mütter im

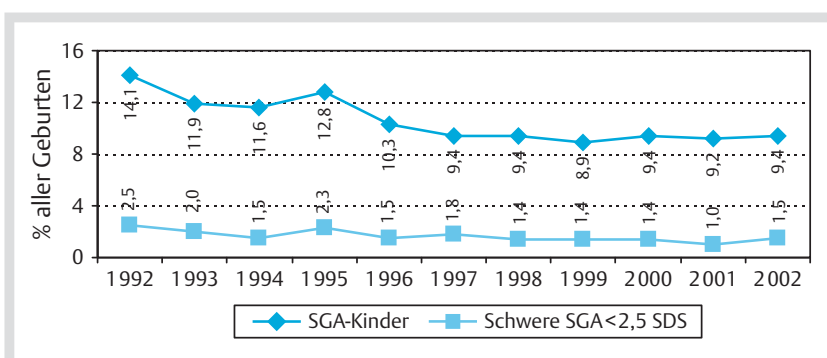

Abb. 1 Anteil retardierter Neugeborener bezogen auf alle Lebendgeburten, SGA - small for gestational age, Geburtsgewicht <1. SDS (10. Perzentile), schwere SGA <2,5 SDS (3. Perzentile).

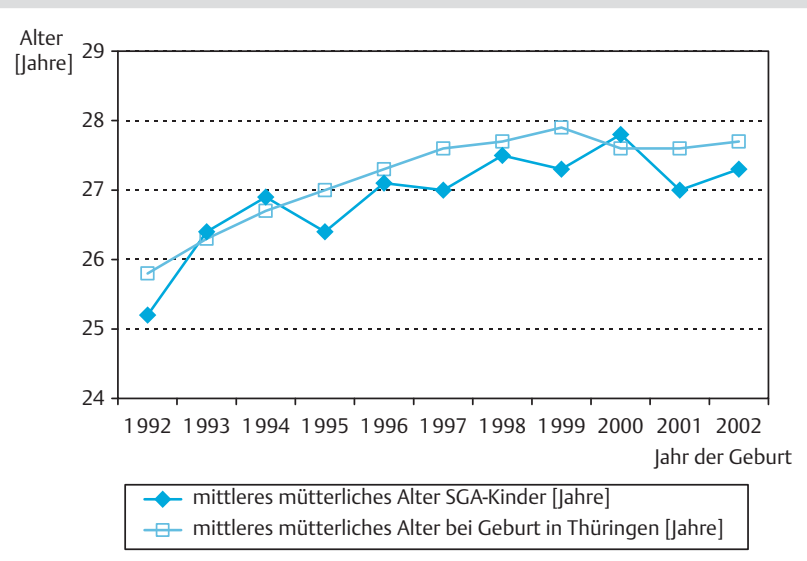

Abb. 2 Alter der Mutter bei Geburt im Zeitraum 1992-2002. 


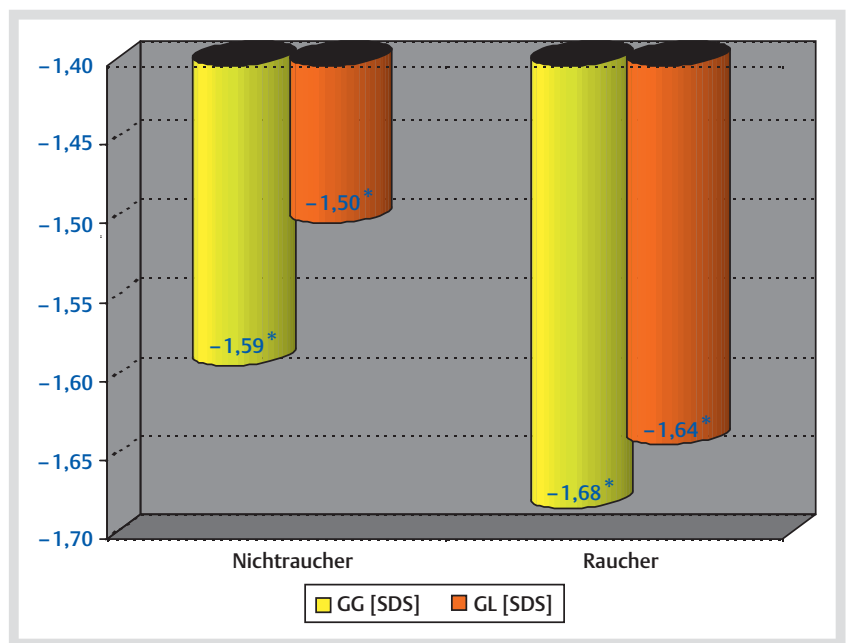

Abb. 3 Geburtsgewicht [SDS] und Geburtslänge [SDS] bei Kindern von Raucherinnen vs. Nichtraucherinnen.

Vergleich zu Nichtrauchern ( $\bullet$ Abb. 3). Im SGA-Kollektiv lag der mittlere Nikotinkonsum bei 2 und bei der Gruppe mit schwer retardierten Kindern bei 3,2 Zigaretten pro Tag (bis maximal 40 Zig./d). Der tägliche Zigarettenkonsum korrelierte im SGA-Kollektiv negativ mit dem GG $(r=-0,09 ; \mathrm{p}<0,01)$ sowie mit der $G L$ $(\mathrm{r}=-0,08 ; \mathrm{p}<0,01)$.

Überraschenderweise fand sich ein signifikanter Unterschied bei der mütterlichen Körpergröße, wobei die rauchenden Mütter im Mittel kleiner als die Nichtraucherinnen waren $(\mathrm{p}<0,05)$. Raucherinnen im untersuchten Kollektiv waren im Durchschnitt 1,2 Jahre jünger als Nichtraucherinnen $(p<0,001)$.

Um Aussagen über das Wachstumsverhalten in der Kindheit zu erhalten wurden 383 Elternpaare angeschrieben, von denen 108 aktuelle Größen- und Gewichtsangaben ihrer Kinder übermittelten, was einem Datenrücklauf von 29,2\% entspricht. Zum Zeitpunkt dieser aktuellen Messung waren die Kinder zwischen 2,5 und 13,5 Jahre alt (im Mittel 8,0 $\pm 3,4$ Jahre).

75 der 108 Kinder (69,4\%) hatten ihren Wachstumsrückstand aufgeholt, während 33 Kinder (30,6\%) mit einer Körpergröße $\leq-2,0$ SDS kein Aufholwachstum zeigten ( $\bullet$ Abb. 4). Bei der Entwicklung des Körpergewichtes zeigte sich, dass alle Kinder ihr zur Geburt bestehendes Gewichtsdefizit ausgeglichen haben. Jedoch fanden sich 8 übergewichtige Kinder (7,4\%) mit einem Körpergewicht zwischen $+1,0$ und $+2,0$ SDS und 5 adipöse Kinder (4,6\%) mit einem Gewicht größer + 2,0 SDS ( $\bullet$ Abb. 5).

Lediglich 70,6\% der Kinder von rauchenden Müttern wiesen ein Aufholwachstum auf, während 85,5\% der Kinder der Nichtraucherinnen mit ihrer aktuellen Körpergröße den Normbereich erreicht hatten. Somit ergibt sich für SGA-Kinder, deren Mütter während der Schwangerschaft rauchten ein doppelt so hohes Risiko (29,4\% vs. $14,5 \%$ ) für das Ausbleiben des Aufholwachstums.

\section{Diskussion}

\section{$\nabla$}

In der Studie wurden die kurz- und langfristigen Auswirkungen eines maternalen Nikotinabusus während der Schwangerschaft in einer Kohorte wachstumsretardiert geborenen Kinder, die von 1992-2002 in 2 Thüringer Perinatalzentren geboren wurden, untersucht. Der Anteil von small-for-gestational-age Kindern im Gesamtkollektiv entspricht mit 10,5\% der für Deutschland zu erwartenden Relation [1].

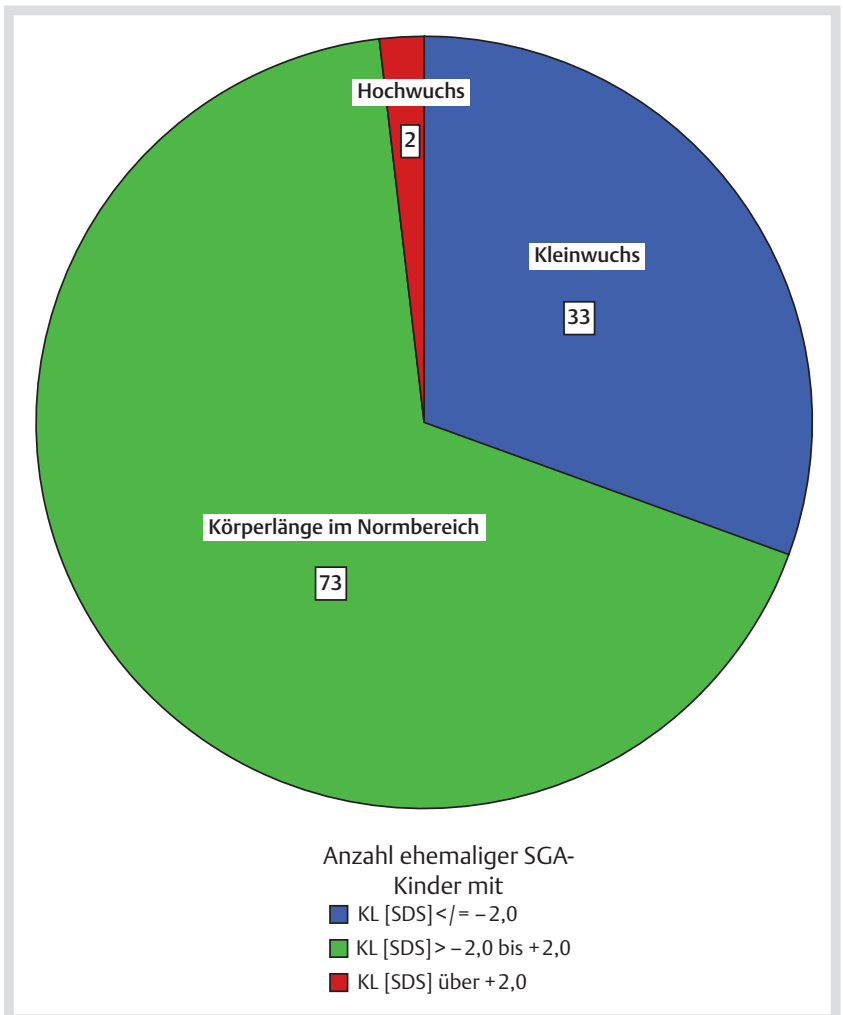

Abb. 4 aktuelle Körpergröße ehemaliger SGA-Kinder $(n=108)$.

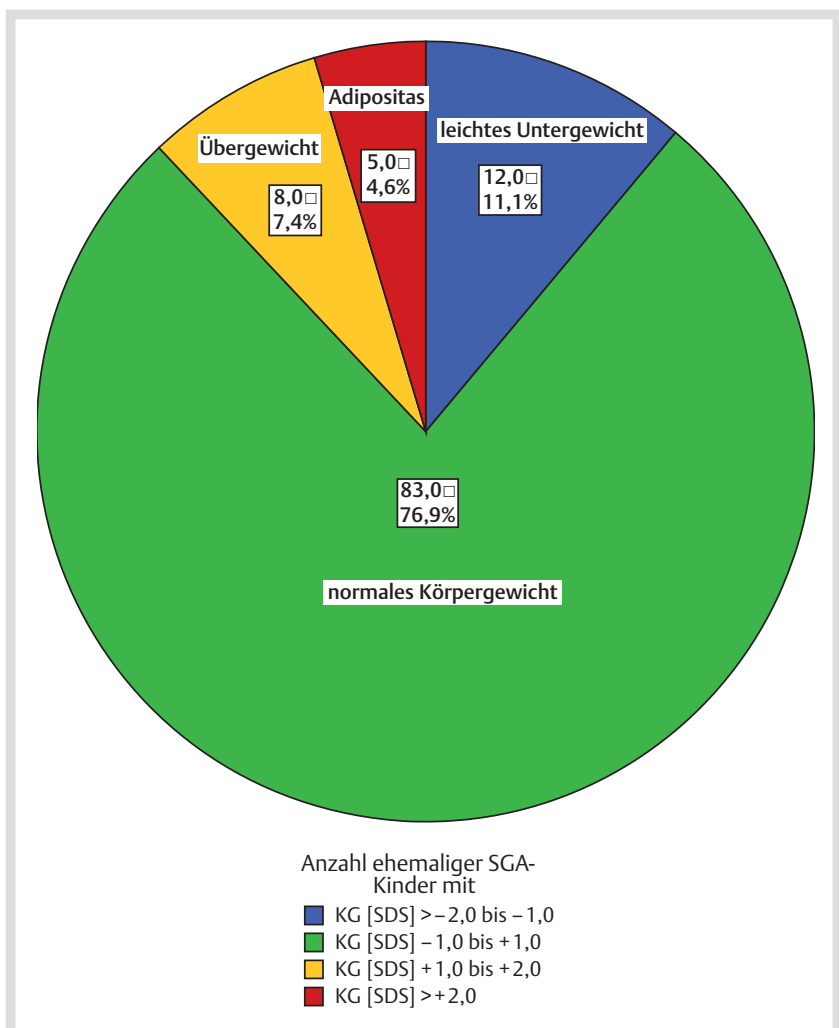

Abb. 5 aktuelles Körpergewicht ehemaliger SGA-Kinder $(n=108)$. 
Während des 11-jährigen Beobachtungszeitraumes reduzierte sich die Zahl der Kinder mit Geburtsmaßen unter der 10. Perzentile um fast 5\%, während sich der Anteil schwer retardiert geborener Kinder nicht wesentlich veränderte. Da in den ersten Jahren des letzten Jahrzehntes die Geburtenrate in Thüringen um über $40 \%$ gesunken ist [8], können demografische Effekt als Ursache der erhöhten Rate wachstumsretardiert geborener Kinder in der ersten Hälfte des Beobachtungszeitraums nicht ausgeschlossen werden. Wie aus der Literatur bekannt war die Frühgeburtlichkeit sowohl im SGA-Kollektiv mit $14 \%$ als auch bei den schwer retardierten Kindern mit 29\% deutlich erhöht [9-11]. Die Geschlechtsverteilung im SGA-Kollektiv war nahezu ausgeglichen, womit sich in unserer Untersuchung kein Einfluss des Geschlechts auf eine intrauterine Wachstumsverzögerung zeigte. In der Gruppe der schwer wachstumsretardierten SGA-Kinder dominierten hingegen 55,9\% Mädchen gegenüber nur 44,1\% Jungen, was durch die höheren Totgeburten- und Mortalitätsraten männlicher Neugeborener bedingt sein könnte [12,13].

Rauchen während der Schwangerschaft wird als ein wesentlicher Risikofaktor für eine Intrauterinen Wachstumsrestriktion seit langem diskutiert und ist in bis zu $40 \%$ mit einer SGA assoziiert [3]. Der auch in unserem Kollektiv gefundene negative Einfluss des Zigarettenkonsums auf die somatischen Geburtsparameter bestätigt die vorhanden Studien $[3,11,14]$. Interessanterweise konnten wir auch einen signifikanten Unterschied für die Körpergröße der Mütter zeigen, wobei die rauchenden Mütter im Durchschnitt kleiner waren als Nichtraucherinnen, der so bisher nicht beschrieben worden war. Eine Erklärung könnte ein niedrigerer sozioökonomischer Status mit einer unausgewogenen Ernährung sein. In der Wessex Growth Study, in der kleinwüchsige Schulanfänger mit unauffälligen Kontrollen verglichen wurden, zeigte sich, dass 31 \% der kleinwüchsigen Kinder aus Familien mit niedrigem sozioökonomischen Status kamen, während dies nur für 13\% der Kinder aus der Kontrollgruppe zutraf [15].

Langfristige Effekte einer pränatalen Nikotinexposition auf das spätere kindliche Wachstumsverhalten konnten anhand der Daten von 108 Kindern, deren Geburtsmaße <2 SDS lagen, erfasst werden. 30,6\% der schwer wachstumsretardiert geborenen Kinder konnten ihren Wachstumsrückstand nicht aufholen. In der Literatur wird dies nur bei $10-20 \%$ der Kinder, die älter als 2 Jahre waren, berichtet [9,16-19]. Bei einer Rücklaufquote von $29 \%$ ist jedoch ein Selektionsbias nicht auszuschließen, da Eltern von kleinwüchsigen Kindern eventuell eher zur Teilnahme an unserer Studie bereit waren. Ein Aufholwachstum bei wachstumsretardiert geborenen Kindern findet hauptsächlich im Verlauf der ersten beiden Lebensjahre statt. Der Grund für ein Ausbleiben des Aufholwachstums ist nicht bekannt. Es wird eine subnormale endogene STH-Sekretion diskutiert [20]. Andere Autoren sehen eine Störung im Bereich der STH-IGF-I-Achse als Hauptgrund und konnten zum Teil erniedrigte IGF-I-Spiegel nachweisen $[8,21,22]$.

Erstmals konnten wir zeigen, dass das Risiko für ein ausbleibendes Aufholwachstum durch eine pränatale Nikotinexposition mehr als verdoppelt wurde. Da in unserer epidemiologischen Studie keine Hormonprofile bestimmt werden konnten, kann über die zur Grunde liegenden Mechanismen keine Aussage getroffen werden.

Eine intrauterine Mangelversorgung ist mit einem erhöhten Risiko für Adipositas, Diabetes mellitus und ein metabolisches Syndrom im späteren Leben verknüpft [2,23]. Diese fetale (Fehl-)
Programmierung der Stoffwechselregulation ist besonders bei Kindern mit einem schnellen Aufholwachstum ausgeprägt. In den von uns untersuchten Kindern hatten alle ihre Gewichtsdefizit nach der Geburt ausgeglichen. $12 \%$ der Kinder wiesen bereits eine Übergewicht oder eine Adipositas auf.

Zusammenfassend kann festgestellt werden, dass der Nikotinkonsum während der Schwangerschaft das Risiko für eine intrauterine Wachstumsrestriktion erhöht und darüber hinaus ein Risikofaktor für eine bleibende Störung der Wachstumsdynamik im späteren Leben zu sein scheint. Es bedarf weiterer endokrinologischer Untersuchungen die zu Grunde liegenden Mechanismen aufzudecken. Alle wachstumsretardiert geborenen Kinder bedürfen einer sorgfältigen Überwachung im Rahmen der kinderärztlichen Vorsorge, um die Kinder mit einem mangelhaften Aufholwachstum frühzeitig diagnostizieren und eventuell therapieren zu können.

\section{Literatur}

1 Voigt M, Friese K, Schneider KTM et al. Kurzmitteilung zu den Perzentilenwerten für die Körpermaße Neugeborener. Geburtshilfe Frauenheilkunde 2002; 62: 274-276

2 Schleußner E. Intrauterine programming der Reproduktiven Funktion - ein valides Konzept? Gynakol Geburtshilfliche Rundsch 2009; 49 (1): $2-7$

3 Wollmann HA. Intrauterine Growth Restriction: Definition and Etiology. Hormone Research 1998; 49 (Suppl 2): 1-6

4 Voigt M, Schneider KTM, Jahrich G. Analyse des Geburtengutes des Jahrgangs 1992 der Bundesrepublik Deutschland. Geburtshilfe Frauenheilkunde 1996; 56: 550-558

5 Lawrence C, Fryer JG, Karlberg $P$ et al. Modelling of reference values for size at birth. Acta Paediatr Scand 1989; 350: Suppl: 55-69

6 Hesse V, Voigt M, Salzler A et al. Alterations in height, weight, and body mass index of newborns, children, and young adults in eastern Germany after German reunification. J Pediatr 2003; 142 (3): 259-262

7 Ziegler R. Endokrinologie - Grundlagen Klinik Praxis. Fischer Verlag Jena; Stuttgart: 1997; 105-131

8 Statistischen Landesamt Thüringen http://www.tls.thueringen.de/ datenbank

9 Mongelli M, Gardosi J. Fetal growth. Curr Opin Obstet Gynecol 2000; 12: $111-115$

10 Tan TYT, Yeo GSH. Intrauterine growth restriction. Curr Opin Obstet Gynecol 2005; 17: 135-142

11 Wollmann HA. Intrauterine Wachstumsretardierung. Monatsschr Kinderheilk 1998; 146: 714-726

12 Hamm W, Göhring UJ, Günther $M$ et al. Geburtshilfliche Prognosefaktoren bei Frühgeborenen sehr niedrigen Geburtsgewichts $(\leq 1500$ Gramm) hinsichtlich Überlebensraten und frühkindlicher Entwicklung. Geburtsh Frauenheilk 1995; 55: 150-155

13 Msall ME, Buck GM, Rogers BT et al. Predictors of mortality, morbidity and disability in a cohort of infants $\leq 28$ weeks' gestation. Clin Pediat 1993; 23: 521-527

14 Nordentoft M, Lou HC, Hansen D et al. Intrauterine growth retardation and premature delivery: the influence of maternal smoking and psychosocial factors. Am J Public Health 1996; 86 (3): 347-354

15 Voss LD, Mulligan J, Betts PR. Short stature at school entry - an index of social deprivation? (The Wessex Growth Study). J Trop Pediatr 1997; 43 (5): 297-303

16 De Zegher F, Hokken-Koelega A. Growth hormone therapy for children born small for gestational age: height gain is less dose dependent over the long term than over the short term. Pediatrics 2005; 115 (4): e458-e462

17 Hokken-Koelega AC, De Ridder MA, Lemmen RJ et al. Children born small for gestational age: do they catch up? Pediat Res 1995; 38 (2): $267-271$

18 Lee PA, Kendig JW, Kerrigan JR. Persistent short stature, other potential outcomes, and the effect of growth hormone treatment in children who are born small for gestational age. Pediatrics 2003; 112 (1): $150-162$

19 Levy-Marchal C, Jaquet D. Long-term metabolic consequences of being born small for gestational age. Pediat Diab 2004; 5 (3): 147-153 
20 De Waal WJ, Hokken-Koelega AC, Stijnen $T$ et al. Endogenous and stimulated GH secretion, urinary GH excretion, and plasma IGF-I and IGF-II levels in prepubertal children with short stature after intrauterine growth retardation. Clin Endocrinol 1994; 41 (5): 621-630

21 De Zegher F, Francois I, Van Helvoirt $M$ et al. Small as fetus and short as child: from endogenous to exogenous growth hormone. J Clin Endocrinol Metab 1997; 82 (7): 2021-2026
22 Deiber $M$, Chatelain $P$, Naville $D$ et al. Functional hypersomatotropism in small for gestational age (SGA) newborn infants. J Clin Endocrinol Metab 1989; 68 (1): 232-234

23 Barker $D$. The Long-term outcome of retarded fetal growth. Clin Obstet Gynecol 1997; 40 (4): 853-863 